\title{
Interplay between chaos and external noise in an extended system: improved forecasting due to intrinsic stochastic resonant phenomena
}

\author{
Jorge A. Revelli, Miguel A. Rodriguez and Horacio S. Wio \\ Instituto de Física de Cantabria, \\ Universidad de Cantabria and CSIC, \\ Avda. Los Castros s/n, E-39005 Santander, Spain
}

\begin{abstract}
We have investigated the effects of noise on an extended chaotic system using as benchmark the Lorenz'96 model. The analysis of the system's time evolution and its time and space correlations gave us numerical evidence for two distinct stochastic resonance-like behaviors, that become apparent when the signal-to-noise ratio is depicted as a function of the external noise intensity, or the system size. Such phenomena result only weakly sensitive to an external periodic signal. The underlying mechanisms can be associated to a noise induced chaos reduction. Resonant phenomena implications in forecasting are studied exploiting a new method that quantifies the reduction of chaos errors' evolution process. A new view of the stochastic resonance phenomenon in a non-stationary situation is shown.
\end{abstract}

Keywords: Spatiotemporal Chaos, Noise, Stochastic Resonance.

\section{INTRODUCTION}

During the last few decades it has been shown that, under certain conditions, the entanglement between nonlinear dynamics and noise can trigger new phenomena that only occur under the effect of such noise: the noise-induced phenomena. A few examples are: stochastic resonance in zero-dimensional and extended systems [Gammaitoni et al., 1998; Lindner et al., 1996; Wio, 1996; Castelpoggi \& Wio, 1997; Castelpoggi \& Wio, 1998; Bouzat \& Wio, 1999; Wio, Bouzat \& von Haeften, 2002; Wio \& Deza, 2007], noise-induced transitions [Horsthemke \& Lefever, 1984], noise-induced phase transitions [Van den Broeck, Parrondo \& Toral, 1994; Van den Broeck et al., 1997; Mangioni et al., 1997; Mangioni et al., 2000], noise-induced transport [Reimann, 2002; Astumian \& Hänggi, 2002; Reimann et al., 1999; Mangioni, Deza \& Wio, 2001], noise-sustained patterns [García-Ojalvo \& Sancho, 1999; von Haeften \& Izús, 2003; Izús G. et al., 2003]; noise-induced limit cycles [Mangioni \& Wio, 2003].

Some of the above indicated noise-induced phenomena occur in spatially extended systems where another phenomena of great relevance usually exists: spatiotemporal chaos [Bohr et al., 1998]. However, studies on the effect of noise on spatially extended chaotic systems are scarce [Gutiérrez, Iglesias \& Rodríguez, 1993; Wang \& Ouyang, 2002; Ambika, Menon \& Harikrishnan, 2006]. There are studies on chaotic systems where the pseudorandom behavior of the system is the trigger of phenomena usually associated with the effect of a real stochastic process (see for instance [Anishchenko, Neiman \& Safanova, 1993; Nicolis, Nicolis \& McKernan, 1993; Anishchenko et al., 1994; Hennig, Schimansky-Geier \& Hänggi, 2007]). Hence, we can refer to the presence of a deterministic noise, that is a pseudo-random behavior associated to the chaotic character of the system.

In a recent paper [Revelli, Rodriguez \& Wio, 2008], the effect of noise on an extended chaotic system was inves- tigated, analyzing the interplay between the above indicated deterministic noise and a real stochastic process. In order to perform such a study the Lorenz'96 model [Lorenz, 1996] was chosen. In spite of the fact that it is a kind of toy-model, and at variance with the cases studied in [Gutiérrez, Iglesias \& Rodríguez, 1993; Wang \& Ouyang, 2002; Ambika, Menon \& Harikrishnan, 2006], it has a clear relevance to real systems as it is of interest for the analysis of climate behavior and weather prediction [Lorenz, 1996; Wilks, 2005; Orrell, 2003]. In fact, this model has been heuristically formulated as the simplest way to take into account certain properties of global atmospheric models. To reach our objective, we have assumed that the only model parameter is time dependent and composed of two parts, a constant deterministic contribution plus a stochastic one. Through the analysis of the temporal evolution of the system and its time and space correlations, numerical evidence was obtained for two stochastic resonance-like (SR) [Gammaitoni et al., 1998] behaviors.

Here we briefly review those results, and also include the analysis of the action of an external periodic signal. We also analyze the effect of SR in forecasting which, in fact, provide us with an alternative procedure suitable to identify the presence of a SR phenomenon in an extended chaotic system. Such a new picture is achieved by exploiting the so called mean-variance of logarithms diagram [López., Primo, Rodríguez \& Szendro, 2004; Gutiérrez, Primo, Rodríguez \& Fernández, 2008].

\section{THE MODEL AND RESPONSE MEASURES}

The equations corresponding to the Lorenz'96 model [Lorenz, 1996; Lorenz \& Emanuel, 1998] are

$$
\begin{gathered}
\frac{d}{d t} x_{j}(t)=-x_{j-1}\left(x_{j-2}-x_{j+1}\right)-x_{j}+F \\
j=1,2,3, \ldots, N .
\end{gathered}
$$


In order to simulate a scalar meteorological quantity extended around a latitude circle, we consider periodic boundary conditions: $x_{0}=x_{N}, x_{-1}=x_{N-1}$. In contrast with other toy models used in the analysis of extended chaotic systems and based on coupled map lattices, the Lorenz'96 system exhibits extended chaos (for $F>F_{t h} \sim 9 / 8$ ), with a spatial structure in the form of moving waves [Lorenz, 1996]. With the appropriated choice of parameters these waves simulate geostrophic eddies.

To take into account external fluctuations we assume that the model parameter $F$ becomes time and space dependent, consisting of two parts, a constant and a random one

$$
F_{j}(t)=F_{0}+\Psi_{j}(t),
$$

with $\Psi_{j}(t)$ a white in space and dichotomic in time process. That is, $\Psi_{j}(t)$ adopts the values $\pm \Delta$, with a transition rate $\gamma$ : each state $( \pm \Delta)$ changes according to the waiting time distribution $\psi_{i}(t) \sim e^{-\gamma t}$. The noise intensity for this process is defined through $\xi=\frac{\Delta^{2}}{2 \gamma}$ [van Kampen, 1992; Gardiner, 1985]. The choice of a dichotomic noise was in order to avoid unnecessary complications as we are dealing with two kinds of noise: a "deterministic" one generated by chaos and an external random one introduced in a parametric way. Since the chaotic noise is quasi-Gaussian, the choice of an external noise of a different kind helped us to distinguish between both effects. However, if necessary, one can take the limit of Gaussian white noise.

As a measure of the SR response of the system we have used the signal-to-noise ratio (SNR) [Gammaitoni et al., 1998]. To obtain the SNR we need to previously evaluate $S(\omega)$, the power spectral density (psd), defined as the Fourier transform of the correlation function [van Kampen, 1992; Gardiner, 1985]

$$
S(\omega)=\lim _{t \rightarrow \infty} \int_{-\infty}^{\infty} e^{-i \omega \tau}\left\langle x_{j}(t) x_{j}(t+\tau)\right\rangle d \tau,
$$

where \langle\rangle indicates the average over realizations of both the external noise and the initial conditions $\left\{x_{j}(0)\right\}$. As we have periodic boundary conditions simulating a closed system, $\left\langle x_{j}(t) x_{j}(t+\tau)\right\rangle$ has a homogeneous spatial behavior. Hence, it is enough to analyze the response at a single site.

In [Revelli, Rodriguez \& Wio, 2008] two forms of SNR were considered. On the one hand, the usual SNR measure at the main resonant frequency $\omega_{0}$ (that is, in fact, at the frequency associated to the highest peak in $S(\omega)$ ). On the other hand, we consider a global form of the SNR $\left(\mathrm{SNR}_{\text {global }}\right)$ defined through

$$
S N R_{\text {global }}=\frac{\int_{\omega_{\min }}^{\omega_{\max }} d \omega S(\omega)}{\int_{\omega_{\min }}^{\omega_{\max }} d \omega S_{\text {back }}(\omega)}
$$

where $\omega_{\min }$ and $\omega_{\max }$ define the frequency range where $S(\omega)$ has a rich peak structure (with several resonant frequencies).
In analogy to previous studies [Gammaitoni et al., 1998], we have identified the background spectrum, $S_{\text {back }}(\omega)$, with one without peaks. This was done using a smooth function joining and fitting the low (left of $\omega_{\text {min }}$ in Fig(1)) and high (right of $\omega_{\max }$ in Fig(1)) frequency values of the psd. We have used a low order polynomial, and have also verified that small changes in the polynomial degree, or the adoption of other smooth functions, do not change the results in any significant way.

The detailed analysis done in [Revelli, Rodriguez \& Wio, 2008], in addition to providing us with strong evidences about the robustness of our results, has shown that both forms of the SNR gives essentially the same results. Hence, for simplicity, in what follows we only use the $S N R_{\text {global }}$ measure.

\section{STOCHASTIC RESONANCE RESULTS}

In [Revelli, Rodriguez \& Wio, 2008] we have analyzed the typical behavior of relative trajectories as $x_{1}(t)-x_{T}$, with $x_{T}$ the time averaged trajectory. When the Lorenz'96 system evolves without external noise (that is $F_{j}(t)$ is constant), its time evolution shows a randomlike behavior, with the main feature that the amplitude of the oscillator does not decay in time. However, when the system is subject to a random force as described in Eq. (2), the temporal response decays, due to the fact that the interaction between the intrinsic evolution and the external noise produces dissipation within the system. Hence the system's time evolution consists of a transitory regime and a stationary one. This was analyzed through the behavior of the "decay" of $\left\langle x_{1}(t)-x_{T}\right\rangle$ (averaging only over noise realizations, with a fixed initial condition). We assumed that this decay can be adjusted by an exponential law, with a decay parameter $(\lambda)$ that only depends on $F_{0}$, but does depends neither on the system size nor on the noise intensity. This analysis is relevant when studying the effects of noise on the stationary regime. From these results it was possible to anticipate, and approximately identify, the existence of two regimes: a weak or undeveloped chaos for $F_{0}<6.0$, and a strong or completely developed chaos for $F_{0}>6.0$. The typical numbers we have used in our simulations are: averages over $10^{3}$ histories, each one with different external noise and initial condition, and $\sim 10^{4}$ simulation time steps (within the stationary regime) with $\Delta t=5 \times 10^{-3}$.

\section{A. System Without External Signal}

We have evaluated $S(\omega)$, the psd, as indicated in Eq. (3). Figure 1-a shows the typical form of the psd $S(\omega)$ for two values of $F_{0}\left(F_{0}=4.5\right.$ and 7.8$)$ and for a noise intensity $\Delta=0.1\left(\xi=5 \times 10^{-4}\right)$. The figure shows a rich peak structure within the interval $0.22<\omega<2.3$. It is worth to comment that the frequencies associated 
with the different peaks approximately correspond to the harmonics of the main (or first) peak frequency.

In Fig. 1-b, we show the form of $S(k)$, the associated spatial spectrum, calculated also as the Fourier transform of the spatial correlation $\left\langle x_{i}(t) x_{i+n}(t)\right\rangle$. Here we depict the spectrum for fixed values of the system's size $(N=256)$, and noise intensity $\left(\Delta=10^{-3}\right.$ and $\left.\gamma=10\right)$, and different values of $F_{0}$. The independence of the position of the peak (indicating a single spatial structure of wavelength $k / 2 \pi=0.2)$ is apparent. However, there is a strong dependence on the peak intensity when varying $F_{0}$, from a net peak for underdeveloped chaos $\left(F_{0}=5\right)$ to a reduced peak for well developed chaos $\left(F_{0}=8\right)$. It is worth remarking here that there is no dependence (or eventually a very weak one) of this behavior on the noise intensity.
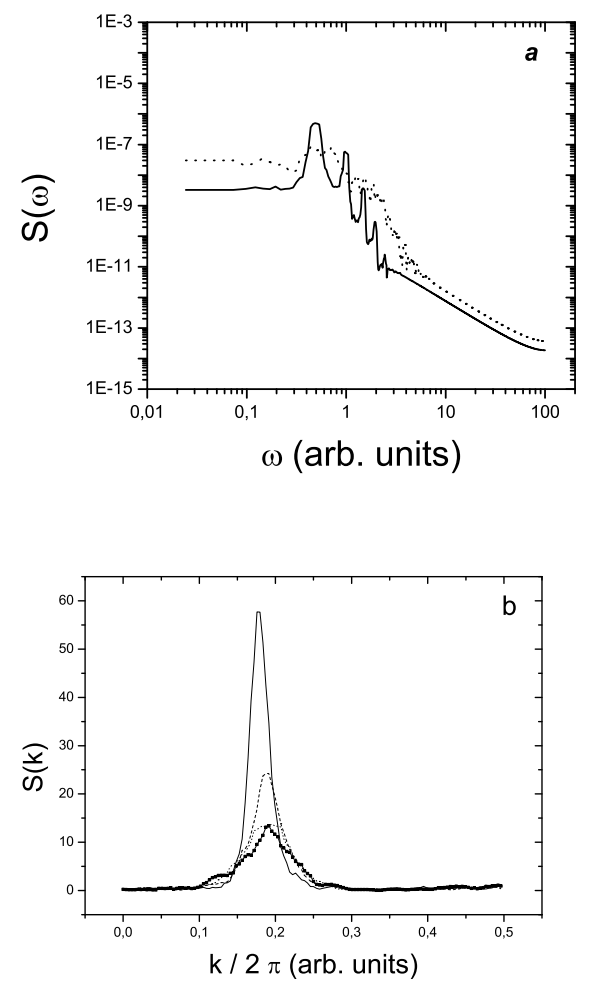

FIG. 1: (a) $S(\omega)$ for a couple of values of $F_{0}$ (dashed line $F_{0}=7.8$, continuous line $F_{0}=4.5$ ) and for a small noise intensity $\left(\xi=5 \times 10^{-4}, \Delta=0.1\right.$ and $\left.N=128\right)$. (b) Spatial spectrum for $N=256, \Delta=10^{-3}$ and $\gamma=10$, for several values of $F_{0}$ : continuous line $F_{0}=5$, dashed $F_{0}=6$, dotted $F_{0}=7$ and dash-dotted $F_{0}=8$.

Figure 2-a shows the dependence of $\mathrm{SNR}_{\text {global }}$-for a space-temporal noise- on $\Delta$ for fixed values of $N$ and two values of $F_{0}$. It shows a peak for $\Delta \sim 6-7 \times 10^{-3}$, that corresponds to the fingerprint of the more usual form of SR.

The analysis of the dependence of $\mathrm{SNR}_{\text {global }}$ on $F_{0}$ has also shown the existence of the previously indicated two regimes: a weak or underdeveloped chaos for $F_{0}<6.0$,
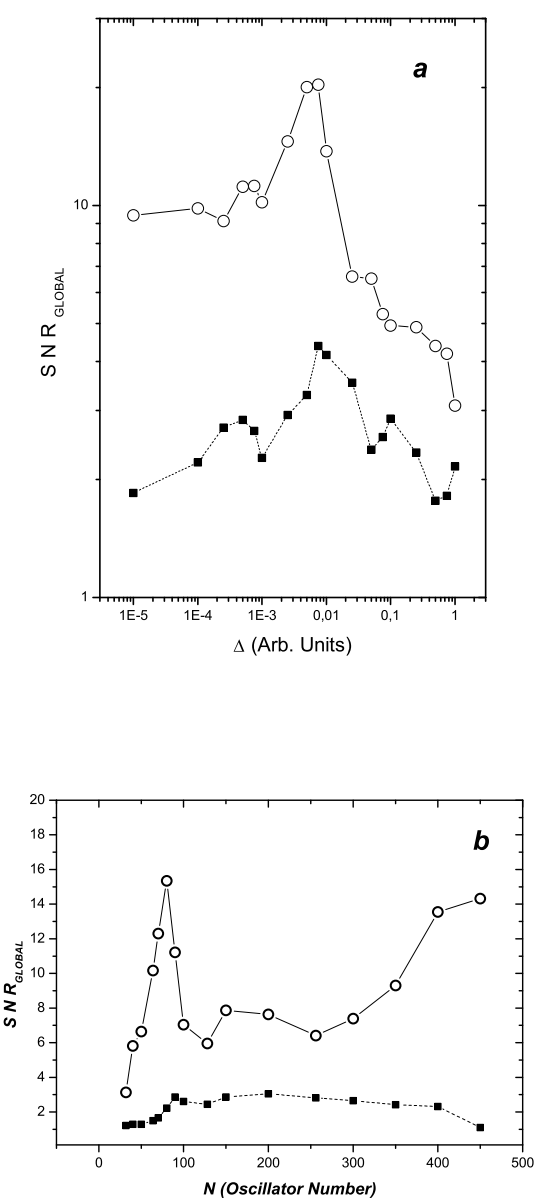

FIG. 2: (a) $\mathrm{SNR}_{\text {global }}$ vs $\Delta$, for fixed values of $N=64$ and $\gamma=10 ;$ and a couple of values of $F_{0}$ : white circles $F_{0}=5$, black squares $F_{0}=6$. (b) $\mathrm{SNR}_{\text {global }}$ vs $N$, for fixed values of $\Delta=0.1$ and $\gamma=5\left(\xi=10^{-3}\right)$, and a couple of values of $F_{0}$ : white circles $F_{0}=5$, black squares $F_{0}=6$.

and a strong or completely developed chaos for $F_{0}>6.0$. These regimes are characterized by the existence of well defined peaks in the psd, in the former case, and a less defined peak structure in the latter, as seen in Fig. 1-a .

In Fig. 2-b we depict the dependence of $\mathrm{SNR}_{\text {global }}$ on $N$, for fixed values of $\Delta=0.1$ and $\gamma=5\left(\xi=10^{-3}\right)$ and two values of $F_{0}$. The existence of the peak at $N \sim 60$ for $F_{0}=5.0$ is apparent. In addition, we observe an increase of $\mathrm{SNR}_{\text {global }}$ for large values of $N$. However, for $F_{0}=6.0$, the peak has disappeared, as has the increase with larger values of $N$. The presence of the peak at $N \sim 60$ is indicative of a kind of system-size stochastic-resonance (SSSR) [von Haeften, Izús \& Wio, 2005].

The figures clearly show that the system's response is much stronger when the system is within the underdeveloped chaos range than when it is in the highly-developed chaos one. Our results also show that the main resonant 
frequency does not depends on the noise intensity, system size, or the correlation rate.

The SR phenomena found here look similar to the so called internal signal SR [Gang et al. 1993; Zhong \& Xin, 2000; Rosenfeld et al., 2001; Pikovsky \& Kurths, 1997]. In previous studies it was shown that in some systems having an internal typical frequency, SR can occur not only at the frequency of an external driving signal, but also at the frequency corresponding to an internal periodic behavior [Gang et al. 1993; Zhong \& Xin, 2000; Rosenfeld et al., 2001]. Figure 3 shows, for fixed values of $F$ and $\gamma$, the behavior of $S(\omega)$ in both cases: with $(\Delta \neq 0)$ and without noise $(\Delta=0)$.

Regarding the mechanism leading to the SR phenomena indicated above, the increase in the SNR is more strongly related to a reduction of the pseudo (or deterministic) noisy background when turning on the real noise, than to the usual reinforcement of the peak height respect to the noisy background at a given frequency. That is, the interplay between "real" and "deterministic" noise conforms to a kind of noise-induced chaos reduction [Balanov, Janson \& McClintock, 2003; Choe et al., 2005; Liao, Yan \& Hou, 2008]. The above indicated reduction trend, as the real noise is turned on, is apparent.

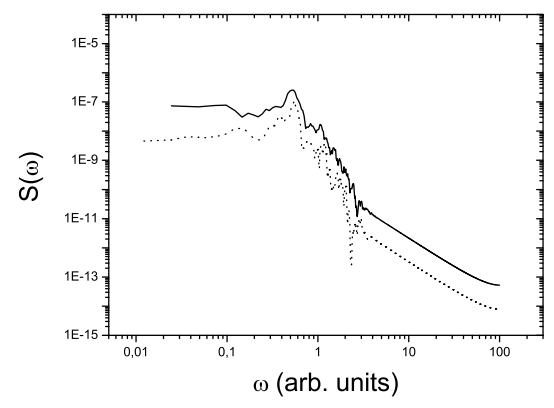

FIG. 3: $S(\omega)$ with and without external noise, for $F=5$ and $\gamma=10$. Continuous line $\Delta=0$, dotted line $\Delta=0.1$.

\section{B. System with External Signal}

In order to consider the effect of an external periodic signal, we assumed that the parameter $F$, in addition to a fluctuating contribution, also includes a periodic (global) one

$$
F_{j}(t)=F_{0}+A \cos [\omega t]+\Psi_{j}(t)
$$

The following figures show the results of considering such a new contribution. In Fig. 4 we depict the dependence of the psd, without noise, for different frequencies and amplitudes. In Fig. 5 we depict $S(k)$, the spatial spectrum for the same cases. Figure 6 shows the behavior of the psd for different noise intensities. From these results it is apparent that the system is almost insensitive to an external frequency.
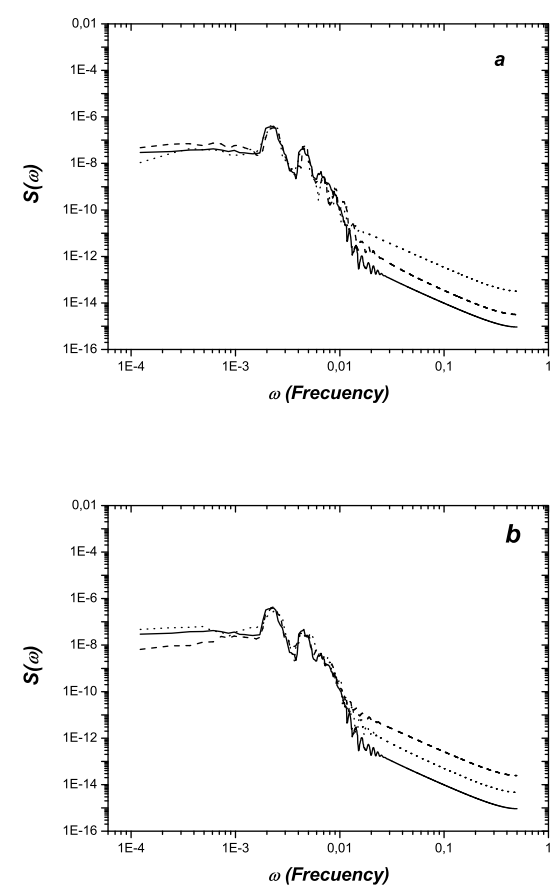

FIG. 4: $S(\omega)$ vs frequency for $F_{0}=4, \Delta=0, N=128$. (a) case $A=0.04$ for different frequencies: continuous line $\omega=10^{-4}$, dashed line $\omega=10^{-3}$, dotted line $\omega=10$. (b) case with $\omega=10^{-4}$, and different amplitudes: continuous line $A=0.04$, dashed line $A=0.2$, dotted line $A=0.4$.

As we will show in the next Section, the indicated SR phenomena are relevant for forecasting. The last results indicate that while the internal signal SR could have a relevant role, the influence of an external periodic seems to be irrelevant from such a point of view.

\section{STOCHASTIC RESONANCE AND FORECASTING}

In order to show the effect of SR on forecasting, we introduce in our toy model a current method used in climate studies, which is based on ensembles.

\section{A. Ensembles Prediction System}

The Ensemble Prediction System (EPS) is one of the most appropriate methodologies to treat uncertainties in the atmospheric models used in forecasting [Molteni, Palmer \& Petroliagis, 1996; Palmer, 2002; Hagedorn \& Doblas-Reyes, 2005]. With this method it is possible to use well known deterministic models in a probabilistic framework [Wang \& Ouyang, 2002; Gottwald \& Mel- 

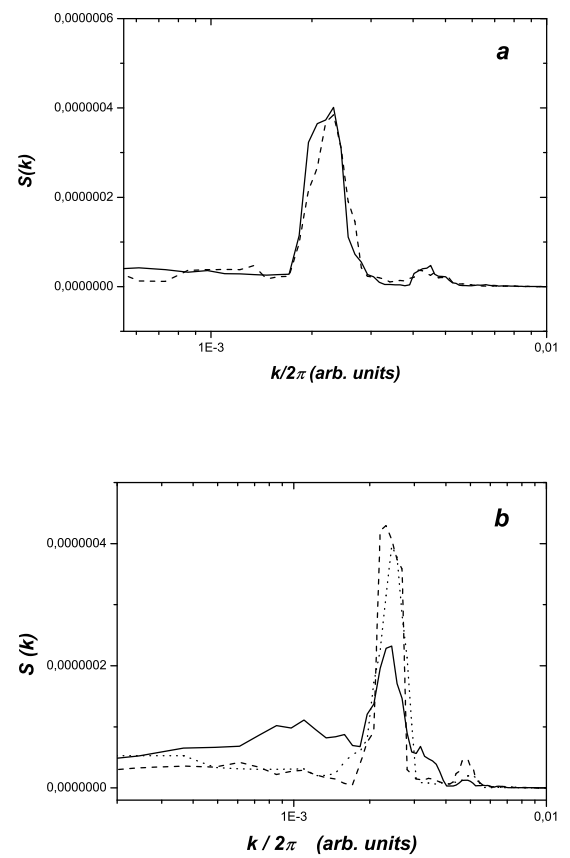

FIG. 5: Space spectral function for $F_{0}=4, N=128$. (a) case $\Delta=0$, continuous line for $A=0.04$ and $\omega=10^{-4}$, dashed for $A=0.8$ and $\omega=10$. (b) case with $\omega=10^{-4}$ and $A=0.4$ and different noise intensities: continuous line $\Delta=10^{-3}$, dashed line $\Delta=10^{-2}$, dotted line $\Delta=10^{-1}$.

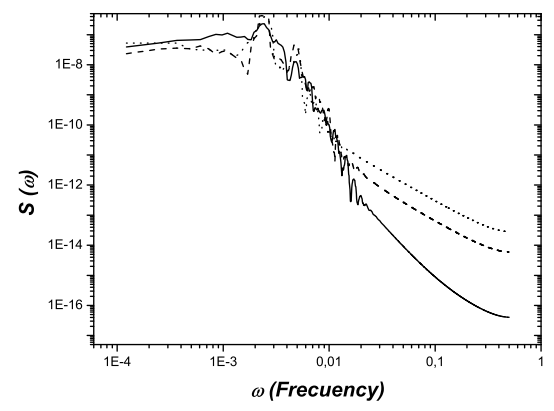

FIG. 6: $S(\omega)$ vs frequency for $F_{0}=4, \omega=10^{-4}, N=128$, and different noise intensities: continuous line $\Delta=10^{-3}$, dashed line $\Delta=10^{-2}$, dotted line $\Delta=10^{-1}$.

bourne, 2005]. A typical system based on ensembles contains a control trajectory and several members of an ensemble. The trajectories of both, the control and the ensemble, belong basically to the same set of data, and both are generated by the model, in our case Eq. (1). At a given time $t_{0}$ we prepare the ensemble by taking its initial condition as a perturbation of the initial condition of the control trajectory $x_{j}^{n}(\tau=0)=x_{j}^{c}(\tau=0) \pm \delta x_{j}^{n}$, $n=1 \ldots N / 2$. Note that we always include two symmetric initial perturbations $\pm \delta x_{j}^{n}$ in order to have a sym- metric ensemble with $N$ members. The evolution of the ensemble (control included) is obtained for several forecast horizons, $\tau=1,2 .$. , using the model with exactly the same realization of noise for each member of the ensemble as well as for the control itself. A set of members $\left\{x_{j}^{n}(\tau)\right\}$ and a control trajectory $x_{j}^{c}(\tau)$ is then obtained. The spreading of the ensemble is analyzed taking as reference the control trajectory $\delta x_{j}^{n}(\tau)=x_{j}^{n}(\tau)-x_{j}^{c}(\tau)$. By definition, $\delta x_{j}^{n}(\tau)$ evolve as finite perturbations.

\section{B. Mean-variance of logarithms diagram}

We introduce now the Mean Variance of Logarithms or $M V L$ diagram [López., Primo, Rodríguez \& Szendro, 2004; Gutiérrez, Primo, Rodríguez \& Fernández, 2008], where the evolution of two indices (indicated below) are displayed along the axis of a two dimensional diagram. It conforms a powerful tool to characterize and graphically represent the evolution of finite perturbations in systems with spatiotemporal chaos, and results to be useful to analyze the dynamics of the differences between each member of the ensemble and the control in an EPS. When the spatial system is chaotic, small initial perturbations show two distinctive characteristics, an exponentially growth of the amplitude, and spatial localization. Both phenomena can be explained as a manifestation of the chaotic effect of stretching and folding in a multidimensional phase space, where each spatial point corresponds to one dimension. Spatial localization arises because there are directions (spatial points) more unstable than others. The difference with a case of low dimensional chaos is that in spatiotemporal chaos these direction are not fixed, and the localization phenomenon becomes dynamic. Apart from the interesting aspect of localization by chaos in the infinitesimal regime, another important phenomenon appears when considering finite perturbations: the progressive loss of correlation due to nonlinear effects that saturates the growth. The $M V L$ diagram shows clearly the two regimes of infinitesimal and finite fluctuations (Fig.7).

Traditionally, only the amplitude growth has been used to characterize a chaotic system through the Lyapunov exponent. In [López, Primo, Rodríguez \& Szendro, 2004] it has been shown how a more complete analysis of finite perturbations requires a knowledge of the spatial localization. The $M V L$ diagram quantifies both aspects of amplitude growth and spatial localization in a simple scheme. It is a simple plot of the first two moments of the logarithm of perturbations. The mean, as expected, is associated with the exponential growth in time. The variance accounts for the spatial localization. These two magnitudes give useful information about different aspects of the dynamics of growth that can be used to characterize ensembles in a prediction system based on them [López., Primo, Rodríguez \& Szendro, 2004; Gutiérrez, Primo, Rodríguez \& Fernández, 2008].

When analyzing perturbations of a system with spa- 


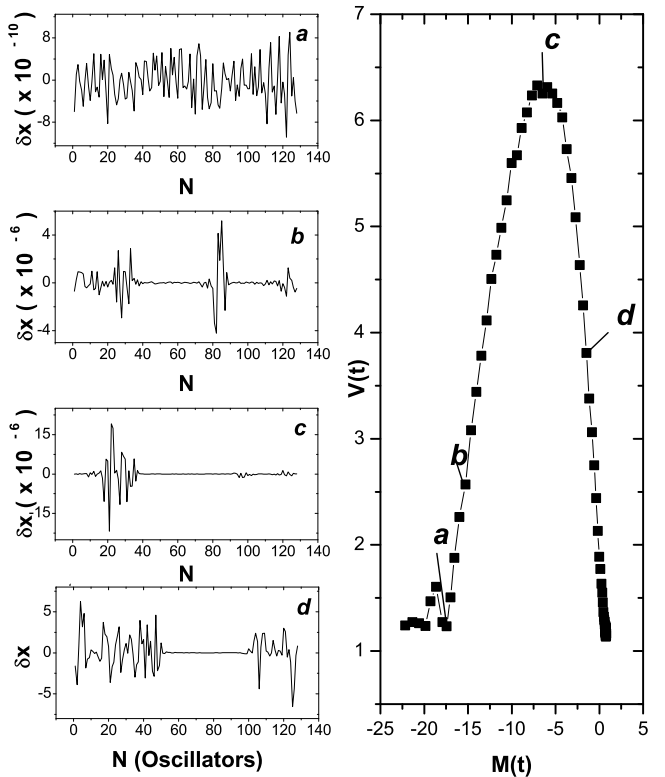

FIG. 7: On the rhs we depict the $M V L$ diagram where four typical times, as indicated in the text, are pointed out. On the lhs we show four snapshots of the system's amplitudes associated to the points indicated on the rhs. It is worth to highlight the different vertical scales in the four cases. Simulations are without noise (perfect model) and the amplitude of the random initial condition is $10^{-4}$

tiotemporal chaos, it is observed that they localize in a fast way due to the multiplicative character of the growth. This results in a log-normal, or in general logbased, statistics. Due to this log-normal characteristic [López, Primo, Rodríguez \& Szendro, 2004], the behavior of the logarithm of absolute values of perturbations

$$
h_{i}(t)=\ln \left(\left|\delta x_{i}(t)\right|\right)
$$

was analyzed. The evolution of this quantity results to be similar to the kinetic roughening of interfaces defined in space $i$ and time $t$ [Barabási \& Stanley, 1995; Pikovsky \& Politi, 1998]. This type of processes can be characterized by using the first two moments, mean and variance (spatial mean is represented by an over-line ... whereas, as before, $\langle\ldots\rangle$ means averaging over samples)

(a) Spatial mean given by

$$
\begin{aligned}
M(t) & =\left\langle\overline{h_{i}(t)}\right\rangle=\left\langle\frac{1}{N} \Sigma_{i} h_{i}(t)\right\rangle \\
& =\left\langle\ln \left(\Pi_{i}\left|\delta x_{i}(t)\right|\right)^{1 / N}\right\rangle=\ln \rho(t)
\end{aligned}
$$

evolves in a characteristic linear regime as $M(t) \sim$ $\ln \rho(0)+\lambda t$ (where $\lambda$ is the leading Lyapunov exponent, see [López, Primo, Rodríguez \& Szendro, 2004] for details).

(b) Width (or roughness) defined as the variance of per- turbations around the spatial mean

$$
V(t)=\operatorname{Var}\left(h_{i}(t)\right)=\left\langle\overline{\left(h_{i}(t)-\overline{h_{i}(t)}\right)^{2}}\right\rangle .
$$

This magnitude grows [López., Primo, Rodríguez \& Szendro, 2004; Gutiérrez, Primo, Rodríguez \& Fernández, 2008] as a power-law of the form $V(t) \sim t^{2 \beta}$ in the linear regime. In the nonlinear regime the variance decays due to nonlinear effects.

The $M V L$ diagram is obtained by plotting $V$ against $M$. In Fig. 7 we show a typical $M V L$ diagram corresponding to the evolution of perturbations $\delta x_{i}$ of the Lorenz'96 model. Here, the initial conditions are random (Gaussian distributed). The evolution goes from left to right, and each point in the diagram corresponds (in a parametric way) to a given forecast horizon. $M(\tau)$ gives the logarithmic amplitude, and is a measure of the ensemble spread on a logarithmic scale. The evolution of the variance of the logarithmic fluctuation $V$, which is analogous to the width of an "effective" interface, follows the typical scaling laws $V(\tau) \sim \tau^{2 / 3}$, behaving as an infinitesimal fluctuation in the first part of the curve. The second part, with a decreasing variance (negative slope), corresponds to the effect of the nonlinearities that progressively destroy the acquired correlation.

In order to offer a clear picture of the growth of the effective interface, we show the effect of the localization on perturbations, as it is the main distinctive effect coming out from chaos. As this phenomenon is far from being intuitive, on the left hand side of the figure we show snapshots of the perturbation space evolution $\delta x_{i}$ for a generic member of the ensemble at four different representative forecasting times.

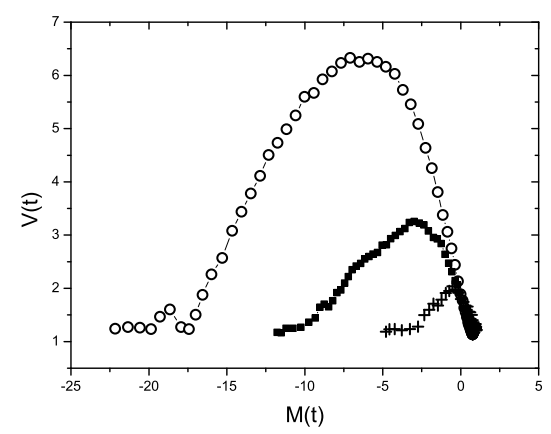

FIG. 8: $M V L$ diagrams for different noise intensities with $\gamma=10$. Open circles $\Delta=0$, black squares $\Delta=10^{-4}$, crosses $\Delta=10^{-1}$. Initial conditions are the same in all three cases, and as in the previous figures. Note that the initial point is not plotted.

Each point (increasing from $(a)$ to $(d)$ ) establishes a typical characteristic evolution time in the $M V L$ diagram. Points $(a)$ and $(b)$ indicate representative times for the system evolution on the curve's positive slope part. That 
is, in the part of the curve where infinitesimal perturbations effects are relevant, with the linearized equations governing the evolution of the system. Point $(c)$ indicates the maximum of the curve, that is the time associated to the maximum localization of infinitesimal perturbations' growth. Finally, point $(d)$, located on the curve's negative slope part, shows a representative time where nonlinear (finite perturbations) effects are relevant, shortly before the system reaches the asymptotic region. From this picture we can see how an increase of $V$ corresponds to a stronger spatial localization in perturbations rather than to an increase in dispersion. This happens in all log-normal (or other log-based) statistical distributions arising from random multiplicative process.

When external noise is added to the ensemble evolution, the $M V L$ diagram change in such a way that the bell-shape form is retained but with a lower amplitude than in the $M V L$ diagram for the case without noise. Hence, we can infer that the bell-shape is due to the chaotic characteristic of the model and the reduction of the bell maximum's high is related to the external noise effect. This is clearly shown in Fig. 8 where the $M V L$ diagram correspond to cases where external noise was added to the evolution of the model. It is worth remarking that the noise does not destroy the analogy with the interface roughening, simply reduces the intensity of the equivalent deterministic noise, as indicated before [Balanov, Janson \& McClintock, 2003; Choe et al., 2005; Liao, Yan \& Hou, 2008].

\section{Detecting SR through the $M V L$}

We have analyzed our system following the methodology indicated in the previous subsection. On Fig. 9-a we show the $M V L$ curves for different noise intensities, while on Fig. 9-b we indicate the maximum of the curves shown on the left part of the figure, as a function of the noise intensity. It is clear that the SR characteristics, that is: a maximum as a function of noise intensity, exists. The variance in the $M V L$ diagram measures the chaos reduction induced by the noise. Figure 10 shows the case of fixed noise intensity and different system's sizes. The "system-size resonance" is apparent, even though the effect seems to be less marked.

One point worth to be pointed out is that, within this approach, both the optimal noise intensity and system's size are shifted respect to the optimal ones in Fig. 2-a,b. This is a consequence that our analysis was performed on a error cycle, that is, in a non-stationary situation. In any case we can conclude that the $M V L$ diagram could be an adequate tool to quantify or detect SR in extended chaotic systems. In order to asset the relevance of this form of analysis, we are performing similar studies in other extended systems where chaos arise. This will the subject of forthcoming papers.
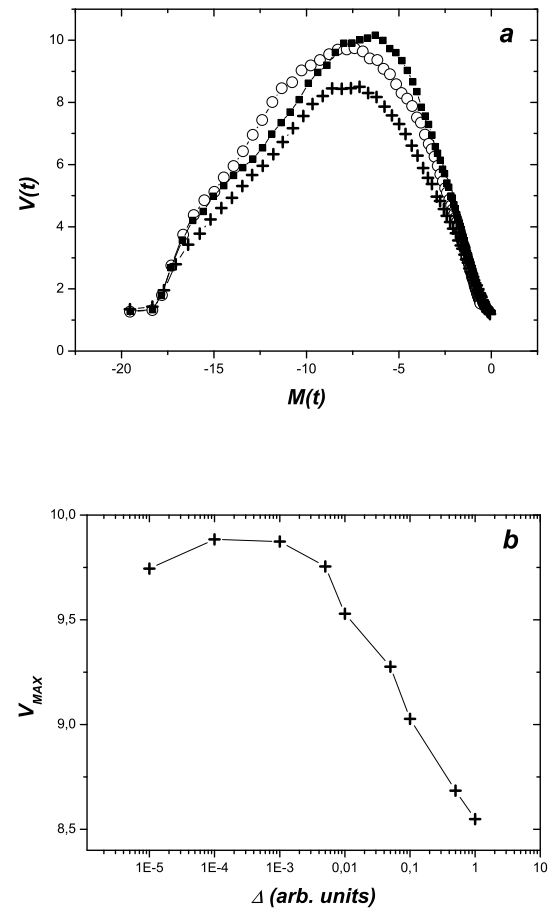

FIG. 9: (a) $M V L$ diagram for different noise intensities with $F_{0}=0.4, A=0.4$ and $\omega=10^{-4}, N=128$ : circles $\Delta=10^{-5}$, black squares $\Delta=10^{-3}$, crosses $\Delta=1$. (b), maximum values of $V$ on the lhs, $V_{\max }$ vs $\Delta$.

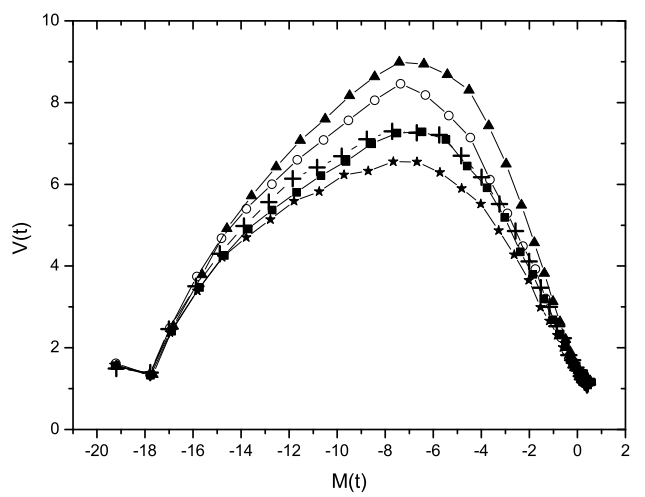

FIG. 10: (a) $M V L$ diagram for different system's size, with $F_{0}=5 ., A=0.4$ and $\Delta=0.1$, as in Fig. 2-b. The different curves correspond to: stars $N=80$,open circles $N=102$, black squares $N=110$, crosses $N=114$, black triangles $N=256$.

\section{CONCLUSIONS}

We have investigated the effect of noise on an extended chaotic system, analyzing the interplay between the deterministic or pseudo-noise and a real random process. For our study we have chosen as benchmark the Lorenz'96 model [Lorenz, 1996] that, in spite of the 
fact of being a toy model, is of interest for the analysis of climate behavior [Wilks, 2005; Orrell, 2003]. We have assumed that $F$, the unique model parameter, is time dependent and composed of two contributions, a deterministic and a stochastic one in a spatiotemporal form.

We have done a thorough analysis of the system's temporal evolution and its time and space correlations. Our results clearly show numerical evidence for two SR-like behaviors. On the one hand a "normal" SR phenomenon occurs at frequencies that seems to correspond to a system's periodic behavior. On the other hand, we have found a SSSR-like behavior, indicating that there is an optimal system size for the analysis of the spatial system's response. As indicated before, the effect of noise is stronger when the chaos is underdeveloped. When analyzing the same system also subject to an external periodic signal, we have found that it is only weakly sensitive to the presence of such and external forcing.

In order to analyze the implications of SR in forecasting, we have introduced a new method to quantified the reduction of chaos in the errors' evolution process. This is achieved exploiting the $M V L$ diagram to measure the chaos reduction induced by the noise, an analysis that was performed on a non-stationary situation. The SR characteristics results apparent when depicting the dynamical evolution using such kind of diagrams. The effect of noise is weak regarding changes in the spatial structure, with the main frequencies remaining unaltered, but it is strong in relation to the strength of the "self-generated" deterministic noise. In fact, in such a system and at the resonant frequencies, forecasting can be improved by the external noise, due to the effect of suppression of the self-generated chaotic noise.

\section{Acknowledgments}

We acknowledge financial support from MEC, Spain, through Grant CGL2007-64387/CLI. JAR thanks the MEC, Spain, for the award of a Juan de la Cierva fellowship. HSW thanks to the European Commission for the award of a Marie Curie Chair during part of the development of this work.

\section{References}

Ambika G., G. Menon and K.P. Harikrishnan, Lattice stochastic resonance in coupled map lattice, Europhys. Lett. 73, 506 (2006).

Anishchenko V.S., A.B. Neiman and M.A: Safanova, Stochastic resonance in chaotic systems, J. Stat. Phys. 70, 183 (1993)

Anishchenko V.S., A. B. Neiman, M.A. Safanova and L.O. Chua, Stochastic resonance in Chua's circuit driven by amplitude or frequency modulated signals, Int. J. Bifurc. \& Chaos 4, 441 (1994).

Astumian A. and P. Hänggi, Brownian Motors, Physics Today 55, 33 (2002).

Balanov AG, Janson NB and McClintock PVE, Coherence resonance of the noise-induced motion on the way to breakdown of synchronization in chaotic systems, Fluct. and Noise Lett. 3 L113-L120 (2003)

Barabási A.L. and Stanley H.E., Fractal Concepts in Surface Growth (Cambridge U.Press, Cambridge, 1995).

Bohr T., M.H. Jensen, G. Paladin and A. Vulpiani, Dynamical Systems Approach to Turbulence, (Cambridge U.P., Cambridge, 1998).

Bouzat S. and H.S. Wio, Stochastic resonance in extended bistable systems: The role of potential symmetry, Phys. Rev. E 59, 5142 (1999)

Castelpoggi F. and H. S. Wio, Stochastic resonance in extended systems: enhancement due to coupling in a reaction-diffusion model, Europhys. Lett. 38, 91 (1997); Castelpoggi F. and H.S. Wio, Stochastic resonant media: Effect of local and nonlocal coupling in reaction-diffusion models, Phys. Rev. E 57, 5112 (1998);

Choe CU, Hohne K, Benner H, et al., Chaos suppression in the parametrically driven Lorenz system, Phys. Rev E 72, 036206 (2005)

Gammaitoni L., P. Hänggi, P. Jung and F. Marchesoni, Stochastic resonance, Rev. Mod. Phys. 70, 223 (1998).

Gang H., T. Ditzinger, C.Z. Ning and H. Haken, Stochastic resonance without external periodic force, Phys. Rev. Lett. 71, 807 (1993)

García-Ojalvo J. and J.M. Sancho, Noise in Spatially Extended Systems, (Springer-Verlag, New York, 1999).

Gardiner C.W., Handbook of Stochastic Methods, 2nd Ed. (Springer-Verlag, Berlin, 1985).

Gottwald G.A. and Melbourne I., Testing for chaos in deterministic systems with noise, Physica D 212, 100 (2005).

Gutiérrez J.M., A. Iglesias and M.A. Rodríguez, Logistic map driven by dichotomous noise, Phys. Rev. E 48, 2507 (1993)

Gutiérrez J.M., Primo C., Rodríguez M.A., and Fernández J., Spatiotemporal characterization of Ensemble Prediction Systems the Mean-Variance of Logarithms (MVL) diagram, Nonlin. Processes Geophys. 15 109-114 (2008).

Hagedorn R., Doblas-Reyes F.J., The rationale behind the success of multi-model ensembles in seasonal forecasting I. Basic concept, Tellus A 57, 219 (2005).

Hennig D., L. Schimansky-Geier and P. Hänggi, Selforganized, noise-free escape of a coupled nonlinear oscillator chain, Europhys. Lett. 78, 20002 (2007).

Horsthemke W. and R. Lefever R., Noise-Induced Transitions: Theory and Applications in Physics, Chemistry and Biology, (Springer, Berlin, 1984).

Izús G., P. Colet, M. San Miguel and M. Santagiustina, Synchronization of vectorial noise-sustained structures, Phys. Rev. E 68, 036201 (2003).

Liao T.L., Yan J.J. and Hou Y.Y., Robust chaos suppression for the family of nonlinear chaotic systems with noise perturbation, Nonlinear Anal.-Theory Meth. \& Applic. 69, 14-23 (2008).

Lindner J.F., et al., Scaling laws for spatiotemporal synchronization and array enhanced stochastic resonance, Phys. Rev. E 53, 2081 (1996). 
López J.M., Primo C., Rodríguez M.A., and Szendro I., Scaling properties of growing noninfinitesimal perturbations in space-time chaos, Phys. Rev. E. 70, 056224 (2004)

Lorenz E.N., The essence of chaos (U.Washington Press, Washington, 1996).

Lorenz E.N. and K.A. Emanuel, Optimal sites for $d u$ plementary weather observation: simulation with a small model, J. Atmosph. Sci. 55, 399 (1998).

Mangioni S., R.R. Deza, H.S. Wio and R. Toral, Disordering Effects of Color in Nonequilibrium Phase Transitions Induced by Multiplicative Noise, Phys. Rev. Lett. 79, 2389 (1997)

Mangioni S., R.R. Deza, R. Toral and H.S. Wio, Phys. Nonequilibrium phase transitions induced by multiplicative noise: Effects of self-correlation, Rev. E 61, 223 (2000).

Mangioni S., R.R. Deza and H.S. Wio, Transition from anomalous to normal hysteresis in a system of coupled Brownian motors: A mean-field approach, Phys. Rev. E 63, 041115 (2001).

Mangioni S. and H.S. Wio, Limit cycle induced by multiplicative noise in a system of coupled Brownian motors, Phys.Rev. E 67, 056616 (2003).

Molteni F., Palmerand T., Petroliagis T., The ECMWF Ensemble Prediction System: Methodology and validation, Q.J. Roy. Meteorol. Soc. 122, 73-119 (1996).

Nicolis G., C. Nicolis and D. McKernan, Stochastic resonance in chaotic dynamics, J. Stat. Phys. 70, 125 (1993)

Orrell D., Model error and predictability over different timescales in the Lorenz'96 systems, J. Atmosph. Sci. 60, 2219 (2003).

Palmer N.T., The economic value of ensemble forecasts as a tool for risk assessment: From days to decades, Q. J. Roy. Meteorol. Soc. 128, 747-774 (2002).

Pikovsky A. and J. Kurths, Coherence resonance in a noise-driven excitable system, Phys. Rev. Lett. 78, 775 (1997). It is a related phenomena, however, it arises in excitable systems, at variance to the usual form of internal signal $S R$.

Pikovsky A. and A. Politi, Dynamic localization of Lyapunov vectors in spacetime chaos, Nonlinearity 11, 1049 (1998).

Reimann P., R. Kawai, C. Van den Broeck and P. Hänggi, Coupled Brownian motors: Anomalous hysteresis and zero-bias negative conductance, Europhys. Lett. 45, 545 (1999)

Reimann P., Brownian motors: noisy transport far from equilibrium, Phys. Rep. 361, 57 (2002).

Revelli J.A., Rodriguez M.A. and Wio H.S., Resonant phenomena in extended chaotic systems subject to external noise: The Lorenz96 model case, Physica A $\mathbf{3 8 7}$. 3103-3110 (2008).

Rosenfeld A.F:, et al., On the influence of noise on the critical and oscillatory behavior of a predatorprey model: coherent stochastic resonance at the proper frequency of the system, Phys. Lett. A 280, 45 (2001).

Van den Broeck C., J.M.R. Parrondo and R. Toral, Noise-Induced Nonequilibrium Phase Transition, Phys. Rev. Lett. 73, 3395 (1994)

Van den Broeck C., J.M.R. Parrondo, R. Toral and R. Kawai, Nonequilibrium phase transitions induced by multiplicative noise, Phys. Rev. E 55, 4084 (1997).

* van Kampen N., Stochastic Processes in Physics and Chemistry (North Holland, Amsterdam, 1992).

von Haeften B. and G. Izús, Noise-sustained structures in differential-flow reactors with autocatalytic kinetics, Phys. Rev. E 67, 056207 (2003)

von Haeften B., G.G. Izús and H.S. Wio, System size stochastic resonance: General nonequilibrium potential framework, Phys. Rev. E 72, 021101 (2005), and references therein.

H. Wang and Q. Ouyang, Effect of colored noises on spatiotemporal chaos in the complex Ginzburg-Landau equation, Phys. Rev. E 65, 046206 (2002);

Wilks D.S., Effects of stochastic parametrizations in the Lorenz'96 system, Quaterly J. of the Royal Meteor. Soc. B 131, 389 (2005).

Wio H.S., Stochastic resonance in a spatially extended system, Phys. Rev. E 54, R3075 (1996)

Wio H.S., S. Bouzat and B. von Haeften, Stochastic resonance in spatially extended systems: the role of far from equilibrium potentials, Physica A 306C, 140 (2002);

Wio H.S. and R.R. Deza, Aspects of stochastic resonance in reaction-diffusion systems: The nonequilibrium potential approach, Europ. Phys. J-Special Topics 146, 111 (2007).

S. Zhong and H. Xin, Internal Signal Stochastic Resonance in a Modified Flow Oregonator Model Driven by Colored Noise, J. Phys Chem. A 104, 297 (2000) 